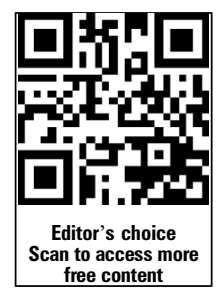

${ }^{1}$ Department of Internal Medicine, University of Utah, Salt Lake City, Utah, USA ${ }^{2}$ Geriatric Research, Education, and Clinical Center, VA Medical Center, Salt Lake City, Utah, USA

${ }^{3}$ Department of Exercise \& Sport Science, University of Utah, Salt Lake City, Utah, USA

${ }^{4}$ Division of Cardiovascular Medicine, Department of Medicine, University of Utah, Salt Lake City, Utah, USA

\section{Correspondence to} Dr D Walter Wray, Department of Internal Medicine, Division of Geriatrics, University of Utah, VAMC SLC, GRECC 182, Bldg. 2 Rm 1D22, 500 Foothill Drive, Salt Lake City, UT 84148, USA; walter.wray@hsc.utah.edu

Received 17 July 2015 Revised 24 August 2015 Accepted 15 October 2015 Published Online First 13 November 2015

\section{SLinked}

- http://dx.doi.org/10.1136/ heartjnl-2015-308852

\section{CrossMark}

To cite: Lee JF, BarrettO'Keefe Z, Garten RS, et al. Heart 2016;102:278-284.

\title{
Evidence of microvascular dysfunction in heart failure with preserved ejection fraction
}

\author{
Joshua F Lee, ${ }^{1,2}$ Zachary Barrett-O'Keefe, ${ }^{2,3}$ Ryan S Garten, ${ }^{1,2}$ Ashley D Nelson, ${ }^{1}$ \\ John J Ryan, ${ }^{4}$ Jose N Nativi, ${ }^{4}$ Russell S Richardson, ${ }^{1,2,3}$ D Walter Wray ${ }^{1,2,3}$
}

\begin{abstract}
Objective While vascular dysfunction is well defined in patients with heart failure (HF) with reduced ejection fraction ( $\mathrm{HFrEF}$ ), disease-related alterations in the peripheral vasculature of patients with $\mathrm{HF}$ with preserved ejection fraction (HFpEF) are not well characterised. Thus, we sought to test the hypothesis that patients with HFpEF would demonstrate reduced vascular function, at the conduit artery and microvascular levels, compared with controls.

Methods We examined conduit artery function via brachial artery flow-mediated dilation (FMD) and microvascular function via reactive hyperaemia $(\mathrm{RH})$ following 5 min of ischaemia in 24 patients with Class II-IV HFpEF and 24 healthy controls matched for age, sex and brachial artery diameter.
\end{abstract}

Results FMD was reduced in patients with HFpEF compared with controls (HFpEF: $3.1 \pm 0.7 \%$; Controls: $5.1 \pm 0.5 \%, p=0.03)$. However, shear rate at time of peak brachial artery dilation was lower in patients with HFpEF compared with controls (HFpEF: 42 070 $4018 / \mathrm{s}$; Controls: $69018 \pm 9509 / s, p=0.01)$, and when brachial artery FMD was normalised for the shear stimulus, cumulative area-under-the-curve (AUC) at peak dilation, the between-group differences were eliminated (HFpEF: $0.11 \pm 0.03 \% / A U C ;$ Controls: $0.09 \pm 0.01 \% / A U C$, $\mathrm{p}=0.58) . \mathrm{RH}$, assessed as AUC, was lower in patients with HFpEF (HFpEF: $454 \pm 35 \mathrm{~mL}$; Controls: $660 \pm 63 \mathrm{~mL}$, $\mathrm{p}<0.01)$.

Conclusions Collectively, these data suggest that maladaptations at the microvascular level contribute to the pathophysiology of HFpEF, while conduit artery vascular function is not diminished beyond that which occurs with healthy aging.

\section{INTRODUCTION}

In many disease states, global reductions in vascular function impact the health of the vascular tree, including conduit vessels and the microcirculation. This is particularly evident in heart failure (HF), a clinical syndrome that presents with numerous symptoms, including impaired cardiac contractile function and systemic vascular dysfunction, which collectively result in the prominent characteristic of severely reduced exercise tolerance and subsequently a reduced quality of life. While HF has classically been characterised by a reduction in ejection fraction, it is now recognised that approximately a half of patients with $\mathrm{HF}$ present with normal or 'preserved' ejection fraction (HFpEF). ${ }^{1}{ }^{2}$ Importantly, the prognosis for HFpEF is similar to that of patients with $\mathrm{HF}$ with reduced ejection fraction (HFrEF), ${ }^{1}{ }^{3}$ yet the vascular pathophysiology of this clinical syndrome remains poorly understood.

Vascular dysfunction has been well documented in patients with $\mathrm{HFrEF}^{4-7}$ In contrast, very few studies have sought to evaluate vascular function in patients with HFpEF, and in the studies that have been undertaken, the results are equivocal. Using a MRI approach, Hundley et $a l^{8}$ reported that flowmediated dilation (FMD) of the superficial femoral artery was similar between patients with $\mathrm{HFpEF}$ and age-matched controls. Subsequent to this, in one of the only studies to assess vascular function using conventional FMD testing, Haykowsky et $a l^{9}$ reported a similar brachial artery FMD in patients with HFpEF compared with healthy, older controls. In contrast, a recent investigation by Farrero et al ${ }^{10}$ demonstrated reduced brachial artery FMD in patients with HFpEF compared with hypertensive controls without HF. Unfortunately, none of these studies appear to have evaluated the shear stimulus that provokes brachial artery FMD, which is viewed as an important consideration to appropriately interpret the vasodilatory response. ${ }^{11}$ Thus, whether patients with HFpEF exhibit vascular dysfunction, as assessed by standardised, up-to-date FMD testing guidelines, ${ }^{12}$ remains uncertain in this patient population.

Though FMD testing has been established as a valuable research tool for non-invasive assessment of vascular function in the conduit vessels, the test provides limited information about vascular function at the level of the microcirculation. Determination of reactive hyperaemia $(\mathrm{RH})$ subsequent to a period of cuff occlusion fills this void, providing an index of microvascular function that is complimentary to conduit vascular function assessed via FMD. There is emerging evidence that $\mathrm{RH}$, assessed via peripheral arterial tonometry (PAT), is reduced in patients with $\mathrm{HFpEF},{ }^{13}{ }^{14}$ and that this disease-related reduction in RH-PAT is independently correlated with incidence of future cardiovascular events ${ }^{15}$ and predictive of poor prognosis. ${ }^{14}$ However, to date, there has not been a study that assessed conduit artery and microvascular function in patients with HFpEF to comprehensively assess peripheral vascular dysfunction in this ever-growing patient population.

Therefore, we sought to determine conduit artery and microvascular function in patients with HFpEF compared with healthy controls using FMD and RH, respectively. We tested the hypothesis that patients with HFpEF would demonstrate 
reduced vascular function, at the conduit artery and microvascular levels, compared with controls.

\section{METHODS \\ Subjects}

Twenty-four patients with Class II-IV HFpEF and 24 healthy control subjects matched for age, sex and brachial diameter volunteered for this study. Patients were recruited from the University of Utah HFpEF Clinic. Within this clinic, patients were screened and included in a manner consistent with the inclusion criteria from the Treatment of Preserved Cardiac Function Heart Failure with an Aldosterone Antagonist (TOPCAT) trial, which included the following criteria: (1) HF defined by the presence of one or more symptoms at the time of screening (paroxysmal nocturnal dyspnea (PND), orthopnoea, dyspnoea on exertion) and one sign (oedema, elevation in jugular venous distention (JVD)) in the previous 12 months; (2) left ventricle ejection fraction $\geq 45 \%$, (3) controlled systolic blood pressure and (4) either one or more hospitalisations in the previous 12 months for which HF was a major component of hospitalisation, or B-type natriuretic peptide in the previous 60 days $\geq 100 \mathrm{pg} / \mathrm{mL}$. Diastolic dysfunction on echocardiogram was diagnosed using a lateral wall $\mathrm{E} / \mathrm{e}^{\prime}$ of $>10$ with a lateral wall $\mathrm{e}^{\prime}$ of $<10$. Exclusion criteria for the HFpEF group included significant valvular heart disease, acute atrial fibrillation and body mass index (BMI) $>45$. All subjects were current non-smokers. The healthy controls were normotensive, free from overt cardiovascular disease, and were not taking any prescription medications. Subjects reported to the laboratory fasted (overnight; $>10 \mathrm{~h}$ ) and had not exercised or consumed caffeine in the $24 \mathrm{~h}$ preceding the study. Data collection occurred in a thermoneutral laboratory with subjects resting in the supine position.

\section{Resting haemodynamic and cardiovascular assessments}

Prior to the FMD and RH tests, supine resting systolic and diastolic arterial blood pressures were determined by an automated blood pressure monitor (Tango+, Suntech, Morrisville, North Carolina, USA) and mean arterial pressure was calculated as diastolic arterial pressure plus a third arterial pulse pressure. Heart rate was monitored from a standard three-lead ECG interfaced with a data acquisition device (Biopac, Goleta, California, USA).

\section{Measurements of brachial artery FMD and RH}

All FMD procedures were performed according to current guidelines. ${ }^{12}$ Following $20 \mathrm{~min}$ of supine rest, baseline measurements of brachial artery diameter and blood velocity were taken for $1 \mathrm{~min}$. Immediately following baseline measurements a blood pressure cuff, placed on the right arm proximal to the elbow and distal to the Doppler probe measurement site, was inflated to a suprasystolic pressure $(>250 \mathrm{~mm} \mathrm{Hg}$ ) for $5 \mathrm{~min}$. The cuff was then rapidly deflated and brachial artery diameter and blood velocity measures were obtained continuously for $2 \mathrm{~min}$. RH was quantified as the cumulative brachial artery blood flow (ie, area-under-the-curve, AUC) for the 2 min period post cuff release.

Blood velocity and vessel diameter were assessed with a Logiq 7 ultrasound Doppler system (GE Medical Systems, Milwaukee, Wisconsin, USA) operating in duplex mode. The Logiq 7 was equipped with a linear array transducer operating at an imaging frequency of $14 \mathrm{MHz}$. Blood velocity was collected with the same transducer at a Doppler frequency of $5 \mathrm{MHz}$ in highpulsed repetition frequency mode $(2-25 \mathrm{kHz})$. Sample volume was optimised in relation to vessel diameter and centred within the vessel. An angle of insonation of $\leq 60^{\circ 16}$ was achieved for all measurements of blood velocity.

\section{Analyses}

Commercially available software (Logiq 7) was used to calculate angle-corrected, time-averaged and intensity-weighted mean blood velocity $\left(\mathrm{V}_{\text {mean }}\right)$. Brachial artery vasodilation was determined offline from end-diastolic, ECG R-wave triggered images collected from the Logiq 7 using automated edge-detection software (Medical Imaging Applications, Coraville, Iowa, USA). Brachial blood flow was calculated based on the formula: brachial blood flow $\left.(\mathrm{mL} / \mathrm{min})=\left(\mathrm{V}_{\text {mean }} \times \pi \text { (vessel diameter } / 2\right)^{2} \times 60\right)$. FMD was quantified using the greatest increase in brachial artery diameter during the 2 min period following cuff release. Shear rate was calculated as: shear rate $(/ \mathrm{s})=8 \quad \mathrm{~V}_{\text {mean }} /$ arterial diameter. Cumulative AUC values for blood flow and shear rate were integrated via the trapezoid rule and calculated as: $\left(\sum(\mathrm{yi}(\mathrm{x}\right.$ $(i+1)-x i)+(1 / 2)(y(i+1)-y i)(x(i+1)-x i)))$.

\section{Blood analysis}

A fasting glucose and lipid panel was performed on blood drawn from an antecubital vein in all subjects using standard methods.

\section{Data analysis and statistical approach}

Statistical analysis was performed with commercially available software (SigmaStat V.3.10, Systat Software, Point Richmond, California, USA). A Student's unpaired $t$ test was used to determine mean differences for subject characteristics, resting mean arterial pressure and heart rate, as well as FMD and RH AUC. A two-way analysis of variance (group $\times$ time) was used to determine differences between groups for $\mathrm{RH}$ following cuff release. When a significant main effect was observed, a Holm-Sidak post hoc analysis was performed. Statistical significance was accepted at $\alpha \leq 0.05$. Group data are presented as mean $\pm S E M$, and exact $\mathrm{p}$ values are given unless otherwise noted.

\section{RESULTS}

\section{Characteristics of patients with HFpEF and age-matched} controls

Anthropometric data and general characteristics for patients and controls are shown in table 1. Disease related characteristics and pharmacological information for the patients with HFpEF are shown in table 2 .

Table 1 Subject characteristics

\begin{tabular}{lccc}
\hline & Controls & HFpEF & p Value \\
\hline Subjects, N (men:women) & $24(10: 14)$ & $24(10: 14)$ & - \\
Age, years & $69 \pm 2$ & $69 \pm 2$ & 0.87 \\
Body mass, kg & $76 \pm 3$ & $101 \pm 6^{*}$ & $<0.001$ \\
Stature, cm & $170 \pm 2$ & $169 \pm 3$ & 0.83 \\
Body mass index, kg/m ${ }^{2}$ & $28 \pm 3$ & $35 \pm 1^{*}$ & 0.03 \\
Body surface area, m & $1.90 \pm 0.04$ & $2.21 \pm 0.07^{*}$ & $<0.001$ \\
Heart rate, beats/min & $62 \pm 2$ & $65 \pm 2$ & 0.25 \\
Mean arterial blood pressure, mm Hg & $98 \pm 2$ & $90 \pm 2^{*}$ & 0.01 \\
Glucose, mg/dL & $84 \pm 2$ & $120 \pm 10^{*}$ & $<0.001$ \\
Cholesterol, mg/dL & $201 \pm 9$ & $163 \pm 10^{*}$ & 0.01 \\
High-density lipoprotein, mg/dL & $51 \pm 3$ & $49 \pm 4$ & 0.98 \\
Low-density lipoprotein, mg/dL & $125 \pm 6$ & $91 \pm 9^{*}$ & $<0.01$ \\
Triglycerides, mg/dL & $149 \pm 17$ & $145 \pm 20$ & 0.89 \\
\hline Data are mean $+S E M$. & & & \\
*Significantly different from Controls. & & & \\
HFpEF, heart failure with preserved ejection fraction. & &
\end{tabular}


Table 2 HFpEF characteristics and medications

\begin{tabular}{|c|c|c|}
\hline \multicolumn{3}{|l|}{ Disease related characteristics } \\
\hline NYHA class II & \multicolumn{2}{|l|}{$10(42 \%)$} \\
\hline NYHA class III & \multicolumn{2}{|l|}{$10(42 \%)$} \\
\hline NYHA class IV & \multicolumn{2}{|l|}{$4(17 \%)$} \\
\hline Six-min walk distance, $\mathrm{m}$ & \multicolumn{2}{|l|}{$402 \pm 42$} \\
\hline B-type natriuretic peptide, $\mathrm{pg} / \mathrm{mL}$ & \multicolumn{2}{|l|}{$150 \pm 26$} \\
\hline Diabetes & \multicolumn{2}{|l|}{$10(42 \%)$} \\
\hline COPD & \multicolumn{2}{|l|}{$2(8 \%)$} \\
\hline CAD & \multicolumn{2}{|l|}{$6(25 \%)$} \\
\hline Hypertension & \multicolumn{2}{|l|}{$20(83 \%)$} \\
\hline Echocardiography & Value & Reference range \\
\hline Ejection fraction, \% & $62 \pm 1$ & $\geq 55$ \\
\hline LV IVSD, cm & $1.1 \pm 0.1$ & $0.6-1.1$ \\
\hline LV PWD, cm & $1.1 \pm 0.1$ & $0.6-0.9$ \\
\hline LV ID diastole, $\mathrm{cm}$ & $4.5 \pm 0.2$ & $3.9-5.3$ \\
\hline LV ID systole, $\mathrm{cm}$ & $3.0 \pm 0.1$ & $2.0-4.0$ \\
\hline Peak E wave, $\mathrm{cm} / \mathrm{s}$ & $84 \pm 7$ & - \\
\hline Peak A wave, $\mathrm{cm} / \mathrm{s}$ & $96 \pm 11$ & - \\
\hline E/A ratio & $1.1 \pm 0.2$ & $0.6-1.32$ \\
\hline $\mathrm{E}^{\prime}$ lateral wall, $\mathrm{cm} / \mathrm{s}$ & $7 \pm 1$ & $13-28$ \\
\hline E/E' ratio & $13 \pm 2$ & $\leq 8$ \\
\hline Mitral E-wave deceleration time, ms & $246 \pm 14$ & $142-258$ \\
\hline \multicolumn{3}{|l|}{ Medications } \\
\hline$\beta$ receptor blocker & $11(46 \%)$ & \\
\hline ACEi or ARB & $15(63 \%)$ & \\
\hline Loop diuretics & $22(92 \%)$ & \\
\hline Aldosterone antagonist & $16(67 \%)$ & \\
\hline Statin & $18(75 \%)$ & \\
\hline Nitrates & $5(21 \%)$ & \\
\hline Calcium channel blockers & $3(13 \%)$ & \\
\hline
\end{tabular}

Data are mean \pm SEM or $\%$ of group.

ACEi, ACE inhibitor; ARB, angiotensin receptor blocker; $C A D$, coronary artery disease; $\mathrm{COPD}$, chronic obstructive pulmonary disease; HFpEF, heart failure with preserved ejection fraction; LV ID, left ventricle internal diameter; LV IVSD, left ventricle interventricular septum diameter; LV PWD, left ventricle posterior wall diameter; NYHA, New York Heart Association.

\section{Brachial artery FMD}

Patients and healthy controls were well matched for baseline brachial artery diameter $(\mathrm{p}=0.44$; table 3$)$. Peak brachial artery diameter following cuff release was not different between groups $(p=0.71)$; however, time to peak was longer in HFpEF compared with controls $(p=0.03$; table 3$)$. Patients with HFpEF demonstrated a reduction in brachial artery FMD, assessed by the peak percentage (HFpEF: 3.06 $\pm 0.68 \%$ (range-5.9-10.7\%, median $=3.03 \%$ ); Controls: $5.06 \pm 0.53 \%$ (range $0.16-10.49 \%$, median $=4.30 \%), \mathrm{p}=0.03$; figure $1 \mathrm{~A}$ ) and absolute (HFpEF: $0.13 \pm 0.03 \mathrm{~mm}$ (range $-0.23-0.35 \mathrm{~mm}$, median $=0.16 \mathrm{~mm}$ ); Controls: $0.23 \pm 0.12 \mathrm{~mm}$ (range $0.01-0.48 \mathrm{~mm}$, median $=0.20$ $\mathrm{mm}), \mathrm{p}=0.02$; figure $1 \mathrm{~B}$ ) change in brachial artery diameter. However, cumulative shear rate at peak dilation was markedly lower in patients compared with controls $(p=0.01$; table 3$)$, and when brachial artery FMD was normalised for shear rate the differences between groups were eliminated for peak percentage (HFpEF: $0.11 \pm 0.03$ (range $-0.14-0.77$, median $=0.10$ ); Controls: $0.09 \pm 0.01$ (range $0.01-0.20$, median $=0.07$ ), $\mathrm{p}=0.58$; figure 2A) and absolute (HFpEF: 0.005 \pm 0.002 (range $-0.008-0.420$, median $=0.004$; Controls: $0.004 \pm 0.001$ (range $0.0005-0.011$, median $=0.003), \mathrm{p}=0.58$; figure $2 \mathrm{~B}$ ) change in brachial artery diameter.
Table 3 Brachial artery flow-mediated dilation

\begin{tabular}{lccc}
\hline & Controls & HFpEF & p Value \\
\hline Baseline brachial artery diameter, mm & $4.43 \pm 0.14$ & $4.61 \pm 0.18$ & 0.44 \\
Peak brachial artery diameter, mm & $4.65 \pm 0.15$ & $4.74 \pm 0.19$ & 0.71 \\
Time to peak dilation, s & $61 \pm 5$ & $78 \pm 6^{*}$ & 0.03 \\
$\begin{array}{l}\text { Cumulative shear rate at time of peak } \\
\text { brachial artery dilation, /s }\end{array}$ & $69018 \pm 9508$ & $42070 \pm 4018^{*}$ & 0.01 \\
\hline $\begin{array}{l}\text { Data are mean } \pm \text { SEM. } \\
\text { *Significantly different from Controls. }\end{array}$ & & \\
HFpEF, heart failure with preserved ejection fraction. & &
\end{tabular}

\section{Reactive hyperaemia}

Although baseline brachial artery blood flow was not different between patients with HFpEF and healthy controls (HFpEF: $86 \pm 9 \mathrm{~mL} / \mathrm{min}$; Controls: $130 \pm 28 \mathrm{~mL} / \mathrm{min}, \mathrm{p}=0.15$; figure $3 \mathrm{~A}$ ), $\mathrm{RH}$ was blunted in patients with $\mathrm{HFpEF}$ during the initial $50 \mathrm{~s}$ following cuff release $(\mathrm{p} \leq 0.03$; figure $3 \mathrm{~A})$. Likewise, $\mathrm{RH}$ assessed as AUC was markedly $(\approx 30 \%)$ lower in patients with HFpEF relative to controls (HFpEF: $454 \pm 35 \mathrm{ml} / \mathrm{min}$ (range $169-798 \mathrm{~mL} / \mathrm{min}$, median $=446 \mathrm{~mL} / \mathrm{min}$ ); Controls: $659 \pm 63 \mathrm{~mL} / \mathrm{min}$ (range $210-798 \mathrm{~mL} / \mathrm{min}$, median=631 $\mathrm{mL} / \mathrm{min}$ ), figure $3 \mathrm{~B})$.

\section{DISCUSSION}

The present investigation sought to comprehensively evaluate peripheral vascular function, at the conduit and microvascular levels, in patients with HFpEF compared with healthy controls. With respect to conduit vessel vascular function, we identified a reduction in brachial artery FMD in patients with HFpEF compared with controls. However, in the HFpEF group, this apparent impairment in FMD was accompanied by a marked $(\approx 40 \%)$ reduction in postocclusion shear rate, a haemodynamic variable that is widely viewed as the stimulus for the FMD response. When FMD was normalised for the shear stimulus, the between-group differences were no longer evident, suggesting an absence of overt vascular dysfunction at the conduit level in patients with HFpEF. In contrast, $\mathrm{RH}$, an index of microvascular function, was significantly $(\approx 30 \%)$ reduced in patients with HFpEF compared with controls, suggestive of abnormal function in the microcirculation in these patients. In combination, these findings provide new evidence in support of the concept that vascular dysfunction in the conduit vessels is not a requisite feature in the pathophysiology of HFpEF, implicating instead a reduction in peripheral microvascular function that may contribute significantly to clinical status and disease progression in this patient group.

\section{Role of the peripheral vasculature in the pathophysiology of HFpEF}

Despite the fact that half of all patients with HF fall into the category of HFpEF, current understanding of the pathophysiology of this pervasive disease remains limited. ${ }^{17}$ The guidelines for clinical diagnosis of HFpEF continue to evolve, with a focus on echocardiographic evidence of abnormal left ventricular relaxation, filling and diastolic stiffness. ${ }^{18}$ However, the classic clinical presentation of HFpEF continues to be defined by dyspnoea upon exertion and severe exercise intolerance, ${ }^{19}$ symptoms that are likely attributable to deficits in cardiac mechanics and peripheral vascular function. ${ }^{20}$ With respect to the latter, a recent study examining 'global cardiovascular reserve' in patients with HFpEF identified a significant correlation between microvascular function $(\mathrm{RH})$ and peak exercise capacity (peak 
Figure 1 Brachial artery flow-mediated dilation (FMD), expressed as per cent (A) and absolute (B) change from baseline in patients with heart failure with preserved ejection fraction (HFpEF) and healthy individuals (Controls). Data are presented as mean \pm SE. *Significantly different from controls, $\mathrm{p} \leq 0.03$.
A

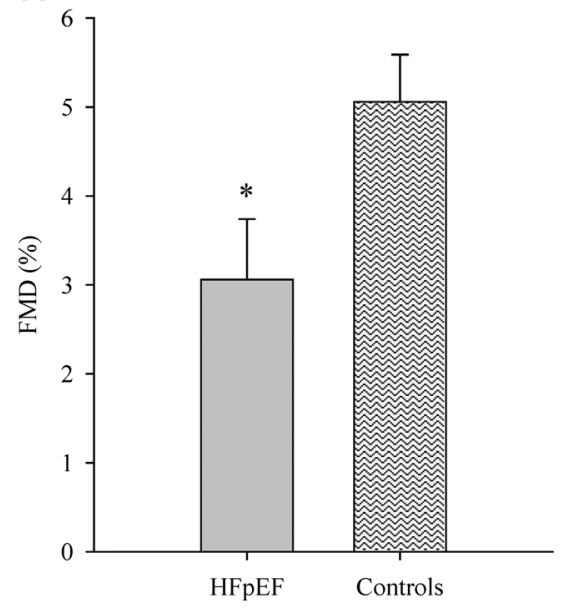

B

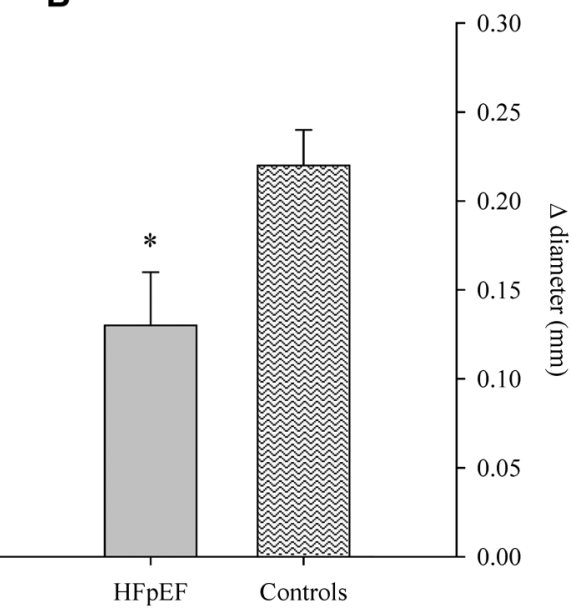

oxygen consumption) and subjective symptoms of fatigue and dyspnoea (Borg scores) during exercise, ${ }^{13}$ highlighting the relationship between peripheral vasculature function and exercise intolerance in this cohort. Further evidence supporting the concept that disease-related changes in the peripheral circulation may contribute significantly to symptom status in HFpEF comes from Dhakal et al, ${ }^{21}$ who recently reported significant reductions in peripheral $\mathrm{O}_{2}$ extraction during incremental cycling exercise in patients with HFpEF compared with HFrEF and control groups that the authors attributed to abnormalities in skeletal muscle or peripheral microvascular function. It is from this framework that the present study was undertaken, with the goal of comprehensively evaluating peripheral vascular function in patients with HFpEF in an effort to better define the vascular pathophysiology in this growing patient group.

\section{Conduit vessel vascular function in $\mathrm{HFpEF}$}

Although vascular dysfunction, assessed by FMD, has been well documented in patients with $\mathrm{HFrEF}^{4-7}$ and is an independent risk factor for an increased risk of clinical events and poor prognosis in this cohort, ${ }^{4}$ much less is known about vascular function in the HFpEF population. In the current study, we observed a reduction in brachial artery FMD in patients with HFpEF compared with controls (figure 1), which initially seemed to indicate impaired conduit vascular function in this patient group. However, assessing the FMD response in this manner does not take into account shear rate, which represents the laminar shear force across the vascular endothelial cells that initiates the FMD response. ${ }^{22}$ The significance of considering this aspect of the stimulus-response relationship should not be underestimated. Indeed, it has been reported that much of the between-subject variability in FMD testing can be significantly reduced by correcting for the shear stimulus, ${ }^{11}$ and current guidelines for FMD testing recommend inclusion of this variable to provide a complete characterisation of the FMD response. ${ }^{12}$ Thus, in view of the substantial reduction in shear rate AUC in patients with $\mathrm{HFpEF}$ at the time of peak vasodilation (table 3), we deemed it appropriate to normalise FMD for wall shear rate. Using this approach, the decrement in FMD observed in the patient group with HFpEF was no longer evident (figure 2), suggesting that the ostensible reduction in \% FMD (figure 1) was, at least in part, the consequence of a reduced shear stimulus.

The current finding that FMD is not attenuated in patients with HFpEF adds to a very small group of studies that have examined conduit vessel vascular function in this cohort. Using a MRI approach, Hundley $e t \mathrm{al}^{8}$ evaluated changes in superficial femoral artery circumference after a 5 min suprasystolic cuff occlusion, and reported that FMD was similar between HFpEF and age-matched controls. Using the more traditional FMD methodology to explore vascular function, Haykowsky et $a l^{9}$ identified similar brachial artery \%FMD in patients with $\mathrm{HFpEF}$ compared with age-matched, healthy controls, which is in contrast to more recent work from Ferrero $e t ~ a l^{10}$ that identified
Figure 2 Brachial artery flow-mediated dilation (FMD) normalised for shear rate area under the curve (SR AUC), expressed as per cent (panel A) and absolute (panel B) change from baseline in patients with heart failure with preserved ejection fraction (HFpEF) and healthy individuals (Controls). Data are presented as mean $\pm \mathrm{SE}$.
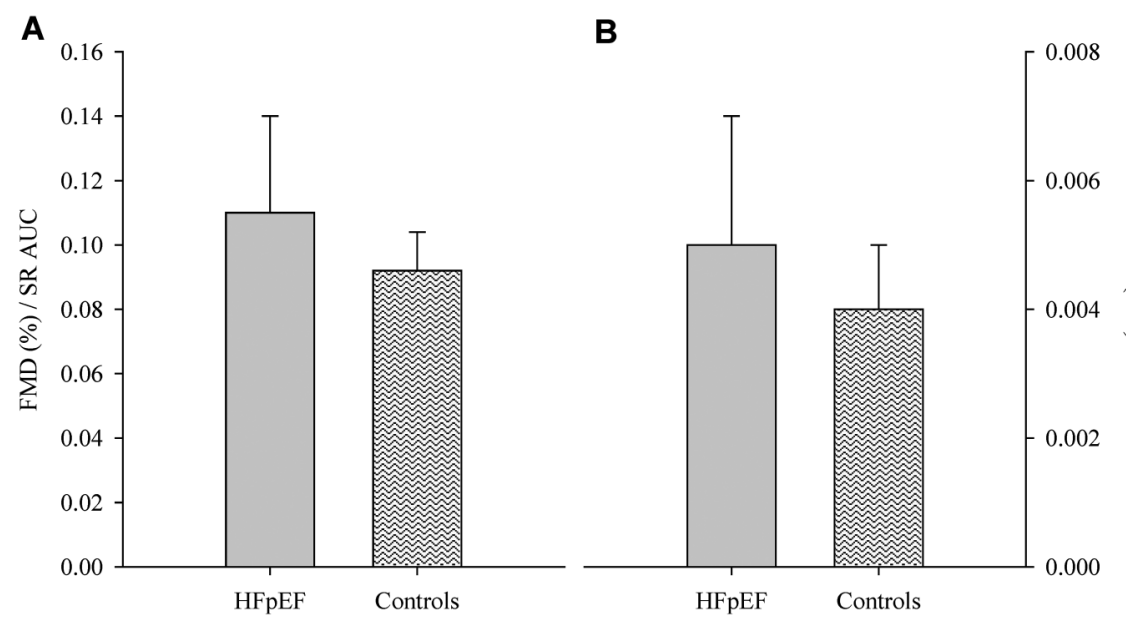
Figure 3 Postocclusion reactive hyperaemia, expressed as absolute blood flow (panel A) and as blood flow area under the curve (AUC; panel $B$ ) in patients with heart failure with preserved ejection fraction (HFpEF) and healthy individuals (Controls). Data are presented as mean \pm SE. *Significantly different from Controls, $\mathrm{p} \leq 0.02$.
A

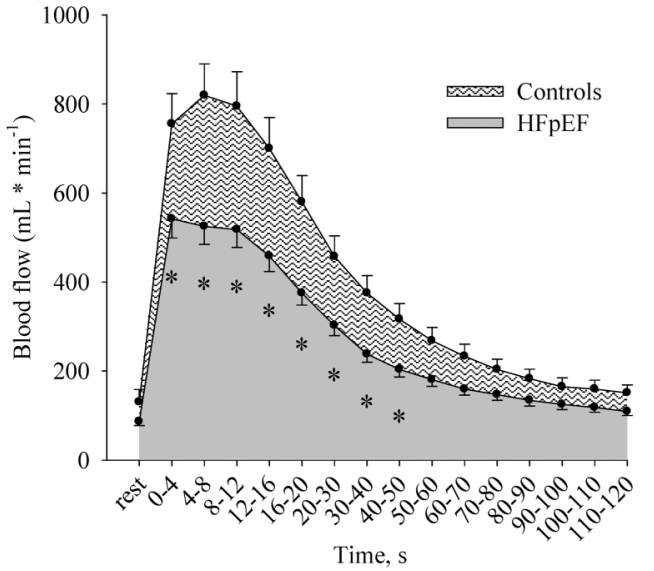

B

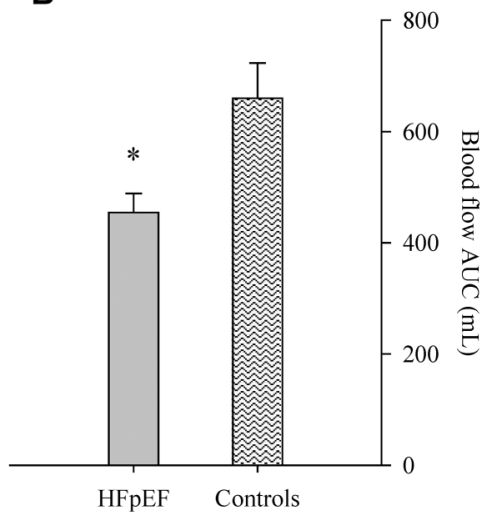

impaired FMD in patients with HFpEF compared with hypertensive controls. With strict adherence to current guidelines regarding FMD testing and careful matching of patients with HFpEF with healthy controls, the present study may be viewed as confirming and extending this previous work, providing new evidence in support of the concept that vascular dysfunction in conduit vessels is not a requisite feature in the pathophysiology of $\mathrm{HFpEF}$.

\section{Microvascular function in HFpEF}

Determination of $\mathrm{RH}$ subsequent to a period of cuff occlusion provides an index of microvascular function that is complimentary to conduit vascular function assessed via FMD. This haemodynamic assessment has been used in a number of patient groups, and has been identified as an independent predictor of prognosis in patients with $\mathrm{HFrEF}^{23}$ and $\mathrm{HFpEF}{ }^{14}$ suggesting that $\mathrm{RH}$ responses may provide important information for risk stratification and determination of disease progression. In the present study, we determined RH by quantifying the blood flow AUC following a 5 min cuff occlusion of the lower arm, and identified a profound $(\approx 30 \%)$ reduction in $\mathrm{RH}$ in patients with HFpEF relative to healthy controls (figure 3 ). This attenuation in the RH response provides clear evidence for the presence of microvascular dysfunction in this patient group that is well beyond the decrement that exists as a consequence of the aging process, which is in agreement with recent work that has used PAT in patients with HFpEF to evaluate $\mathrm{RH}$. Indeed, a recent study by Borlaug et $a l^{13}$ reported a clear reduction in $\mathrm{RH}$ index in patients with $\mathrm{HFpEF}$ and hypertension compared with healthy age-matched controls, which the authors interpreted as evidence of endothelial dysfunction in these patient groups. Using the more conventional forearm RH technique, findings from the present study build on this previous report, adding additional evidence indicating a clear reduction in microvascular function in patients with HFpEF compared with older, healthy controls.

The observed decrement in peripheral microvascular function in HFpEF may be of particular relevance in the context of the coronary circulation, as there is recent evidence implicating coronary microvascular inflammation as a key player in the aetiology of HFpEF. Paulus and Tschope ${ }^{24}$ identified a new paradigm for the aetiology of myocardial remodelling and dysfunction in HFpEF that relates inflammation, perhaps as a consequence of comorbidities such as diabetes, to the subsequent production of reactive oxygen species and reduction in nitric oxide (NO) bioavailability in the coronary microcirculation. According to this proposed model, a shift in myocardial redox balance creates an environment that promotes cardiomyocyte hypertrophy and increased collagen deposition, with the functional consequence of diastolic left ventricular dysfunction. In the same manner that flow-mediated vasodilation in the brachial artery has been demonstrated to correlate with coronary artery vasomotor responses, ${ }^{25}$ it is tempting to speculate that the observed impairment in peripheral microvascular function may provide a surrogate measure for disease-related changes in the coronary microcirculation, and as such, serve as a diagnostic biomarker in this patient group. Additional studies are needed to explore this intriguing possibility.

\section{The distinct vascular pathology of HFpEF}

The present findings identifying dysfunction at different sites along the arterial tree helps to further characterise vascular dysfunction in this cohort, and presents another aspect of the HFpEF pathophysiology that differs significantly from HFrEF. Our group has recently demonstrated that patients with HFrEF have reduced FMD, but not post occlusion RH, compared with healthy age-matched controls. These data suggest that, in contrast to our current findings in HFpEF, conduit artery impairments alone contribute to the vascular dysfunction in HFrEF. This discrepancy between disease-related changes in the conduit and microvascular segments of the peripheral circulation may partially explain why many of the therapeutic approaches that have proven so successful in patients with HFrEF are somewhat less efficacious in the patient group with HFpEF. Indeed, previous studies in HFrEF have identified the ability of drugs targeting the renin-angiotensin-aldosterone system and HMGcoenzyme A reductase inhibition to improve conduit vascular function. ${ }^{2627}$ In contrast, the majority of clinical trials targeting these pathways in HFpEF have proven unsuccessful, ${ }^{28-30}$ though it should be noted that vascular function was not a primary end point in these studies. Thus, while the disparate response to pharmacological treatment is likely due to a host of factors that differ between HFrEF and HFpEF, the distinct manner in which vascular dysfunction manifests in these two cohorts may be worthy of consideration as clinical care of HFpEF continues to evolve.

\section{Experimental considerations}

In the present study, we enrolled patients with HFpEF on optimised pharmacotherapy, and no medications were withheld on experimental days. Thus, we cannot exclude the possibility that existing drug therapy may have affected our measurements of 
vascular function. We also enrolled patients without regards to existing comorbidities such as hypertension, diabetes and coronary artery disease. While this approach may introduce some heterogeneity in terms of baseline parameters, it provided an opportunity to study the pathophysiology of HFpEF in a manner that fairly represents the diverse nature of this patient population. It is acknowledged that the sample size of the present study was relatively small, though a sufficient number of subjects were enrolled to achieve adequate statistical power in the major variables. Finally, we recognise the known variability that is associated with FMD testing, an issue that was somewhat mitigated by the use of standardised testing procedures that conformed to current published guidelines. ${ }^{12}$

\section{CONCLUSIONS}

This investigation documents attenuated vascular function in the microcirculation despite an apparent preservation of conduit artery function in patients with HFpEF compared with wellmatched, healthy controls. These novel findings highlight a specific site of peripheral vascular dysfunction in this patient group that further characterises this disease.

\section{Key messages}

\section{What is already known on this subject?}

Vascular function in the peripheral circulation, assessed by flow-mediated dilation (FMD), has been well documented in patients with heart failure (HF) with reduced ejection fraction (HFrEF). However, there is currently no consensus regarding the presence of vascular dysfunction in patients with $\mathrm{HF}$ with preserved ejection fraction (HFpEF).

\section{What might this study add?}

By investigating vascular function in patients with $\mathrm{HFpEF}$ at the conduit and microvascular levels, we have identified a distinct pattern of vascular dysfunction that is specific to the microvasculature. Brachial artery FMD normalised for shear rate was similar between patients with HFpEF and controls, suggesting an absence of overt vascular dysfunction at the conduit level in this cohort. In contrast, reactive hyperaemia, an index of microvascular function, was significantly $(\approx 30 \%)$ reduced in patients with HFpEF compared with controls, indicative of abnormal function in the peripheral microcirculation.

\section{How might this impact on clinical practice?}

The observed discrepancy between disease-related changes in the conduit and microvascular segments of the peripheral circulation may partially explain why many of the therapeutic approaches that have proven so successful in patients with HFrEF are somewhat less efficacious in the patient group with $\mathrm{HFpEF}$. The results from this study may thus serve to guide the ongoing development of diagnostic algorithms and biomarkers related to vascular function in this patient group.

Contributors All authors contributed to study design, implementation and manuscript preparation.

Funding Funded in part by NIH P01 HL091830, VA RR\&D I01RX000182, VA RR\&D I21 RX001433, VA RR\&D I21 RX001418 and NIH R01 HL118313.

Competing interests None declared.

\section{Patient consent Obtained.}

Ethics approval University of Utah and Salt Lake City Veterans Affairs Medical Center Institutional Review Boards.

Provenance and peer review Not commissioned; externally peer reviewed.

\section{REFERENCES}

1 Bhatia R, Tu J, Lee $D$, et al. Outcome of heart failure with preserved ejection fraction in a population-based study. N Engl J Med 2006;355:260-9.

2 Bursi $F$, Weston $S$, Redfield $M$, et al. Systolic and diastolic heart failure in the community. JAMA 2006;296:2209-16.

3 Owan $T$, Hodge $D$, Herges $R$, et al. Trends in prevalence and outcome of heart failure with preserved ejection fraction. N Engl J Med 2006;355:251-9.

4 Fischer D, Rossa S, Landmesser $U$, et al. Endothelial dysfunction in patients with chronic heart failure is independently associated with increased incidence of hospitalization, cardiac transplantation, or death. Eur Heart J 2005;26:65-9.

5 Katz SD, Biasucci L, Sabba C, et al. Impaired endothelium-mediated vasodilation in the peripheral vasculature of patients with congestive heart failure. J Am Coll Cardiol 1992;19:918-25.

6 Kubo SH, Rector TS, Bank AJ, et al. Endothelium-dependent vasodilation is attenuated in patients with heart failure. Circulation 1991;84:1589-96.

7 Witman MAH, Fjeldstad AS, McDaniel J, et al. Vascular function and the role of oxidative stress in heart failure, heart transplant, and beyond. Hypertension 2012;60:659-68

8 Hundley WG, Bayram E, Hamilton CA, et al. Leg flow-mediated arterial dilation in elderly patients with heart failure and normal left ventricular ejection fraction. Am J Physiol Heart Circ Physiol 2007:292:H1427-34.

9 Haykowsky MJ, Herrington DM, Brubaker PH, et al. Relationship of flow-mediated arterial dilation and exercise capacity in older patients with heart failure and preserved ejection fraction. J Gerontol A Biol Sci Med Sci 2013;68:161-7.

10 Farrero $\mathrm{M}$, Blanco I, Batlle $\mathrm{M}$, et al. Pulmonary hypertension is related to peripheral endothelial dysfunction in heart failure with preserved ejection fraction. Circ Heart Fail 2014;7:791-8.

11 Pyke KE, Tschakovsky ME. The relationship between shear stress and flow-mediated dilatation: implications for the assessment of endothelial function. J Physiol 2005;568:357-69.

12 Harris RA, Nishiyama SK, Wray DW, et al. Ultrasound assessment of flow-mediated dilation. Hypertension 2010;55:1075-85.

13 Borlaug BA, Olson TP, Lam CS, et al. Global cardiovascular reserve dysfunction in heart failure with preserved ejection fraction. J Am Coll Cardiol 2010;56:845-54.

14 Matsue $Y$, Suzuki M, Nagahori W, et al. Endothelial dysfunction measured by peripheral arterial tonometry predicts prognosis in patients with heart failure with preserved ejection fraction. Int J Cardiol 2013;168:36-40.

15 Akiyama E, Sugiyama S, Matsuzawa Y, et al. Incremental prognostic significance of peripheral endothelial dysfunction in patients with heart failure with normal left ventricular ejection fraction. J Am Coll Cardiol 2012;60:1778-86.

16 Logason K, Barlin T, Jonsson ML, et al. The importance of Doppler angle of insonation on differentiation between $50-69 \%$ and $70-99 \%$ carotid artery stenosis. Eur J Vasc Endovasc Surg 2001;21:311-13.

17 Borlaug BA. The pathophysiology of heart failure with preserved ejection fraction. Nat Rev Cardiol 2014;11:507-15.

18 Wachter R, Edelmann F. Diagnosis of heart failure with preserved ejection fraction. Heart Fail Clin 2014;10:399-406.

19 Kitzman DW, Little WC, Brubaker PH, et al. Pathophysiological characterization of isolated diastolic heart failure in comparison to systolic heart failure. JAMA 2002;288:2144-50.

20 Borlaug BA. Mechanisms of exercise intolerance in heart failure with preserved ejection fraction. Circ J 2014;78:20-32.

21 Dhakal BP, Malhotra R, Murphy RM, et al. Mechanisms of exercise intolerance in heart failure with preserved ejection fraction: the role of abnormal peripheral oxygen extraction. Circ Heart Fail 2015;8:286-94.

22 Melkumyants AM, Balashov SA, Khayutin VM. Endothelium dependent control of arterial diameter by blood viscosity. Cardiovasc Res 1989;23:741-7.

23 de Berrazueta JR, Guerra-Ruiz A, Garcia-Unzueta MT, et al. Endothelial dysfunction, measured by reactive hyperaemia using strain-gauge plethysmography, is an independent predictor of adverse outcome in heart failure. Eur $J$ Heart Fail 2010:12:477-83.

24 Paulus WJ, Tschope C. A novel paradigm for heart failure with preserved ejection fraction: comorbidities drive myocardial dysfunction and remodeling through coronary microvascular endothelial inflammation. J Am Coll Cardiol 2013;62:263-71.

25 Anderson TJ, Uehata A, Gerhard MD, et al. Close relation of endothelial function in the human coronary and peripheral circulations. J Am Coll Cardiol 1995;26: 1235-41

26 Varin $\mathrm{R}$, Mulder $\mathrm{P}$, Tamion $\mathrm{F}$, et al. Improvement of endothelial function by chronic angiotensin-converting enzyme inhibition in heart failure : role of nitric oxide, prostanoids, oxidant stress, and bradykinin. Circulation 2000;102:351-6. 
27 Tousoulis D, Oikonomou E, Siasos G, et al. Dose-dependent effects of short term atorvastatin treatment on arterial wall properties and on indices of left ventricular remodeling in ischemic heart failure. Atherosclerosis 2013;227:367-72.

28 Yusuf S, Pfeffer MA, Swedberg K, et al. Effects of candesartan in patients with chronic heart failure and preserved left-ventricular ejection fraction: the CHARM-Preserved Trial. Lancet 2003;362:777-81.
29 Massie BM, Carson PE, McMurray JJ, et al. Irbesartan in patients with heart failure and preserved ejection fraction. N Engl J Med 2008;359: 2456-67.

30 Fu M, Zhou J, Sun A, et al. Efficacy of ACE inhibitors in chronic heart failure with preserved ejection fraction--a meta analysis of 7 prospective clinical studies. Int $J$ Cardiol 2012;155:33-8. 